which illustrates a similar posterior parietal focus ("par.") in flat maps. We used the Montreal Neurological Institute Automated Linear Registration Package [described in D. L. Collins, P. Neelin, T. M. Peters, A. C. Evans, J. Comp. Assist. Tomogr. 18, 192 (1994) and available for free download at ftp://ftp.bic.mni. mcgill.ca/pub/mni_autoreg/] to generate Talairach transformation matrices. We measured the average Talairach coordinates of the center of the parietal focus in the right hemisphere across $n=22$ scan sessions ( $n=12$ unique participants) (Talairach coordinates were as follows: $x=24 \mathrm{~mm}, y=-65 \mathrm{~mm}$, $z=53 \mathrm{~mm}$; SD: $x=6 \mathrm{~mm}, y=5 \mathrm{~mm}, z=6 \mathrm{~mm}$ )

33. Data from both phase-encoded and two-condition experiments were initially analyzed by a fast Fourier transform on the brightness time course from each voxel. Statistical significance was calculated and displayed by converting the Fourier magnitude of the response to an $F$ statistic. When color (red, blue, or green) is used to represent phase, the significance controls the saturation; when color is used to represent significance (red-orange or blue-cyan), the phases are collapsed into two bins (that is, contralateral/ ipsilateral and ON/OFF). The minimal regions of the ipsilateral phase (for example, small blue spots in the F-statistic map in Fig. 3, upper right) have been suppressed in the phase maps (compare the images in Fig. 3, upper left and upper right pairs). The exceptional systematicity of the parietal maps is best appreciated in an animation where each response phase contour is successively marked with a white stripe. Mpeg movies posted at http://kamares. ucsd.edu/ sereno/LIP/ show the response of the left hemisphere (lefthemi.mpg) and the right hemisphere (righthemi.mpg) to one complete target-angle cycle in task 1 on a medial-posterior view of the inflated cortical surface. The relation between the left and right hemisphere responses is illustrated in a closeup movie of the left and right parietal foci (bothcloseup+stim.mpg) that also includes a representation of the corresponding location of the target. In all movies, ipsilateral phases have not been truncated. A small amount of bilateral activation is visible in the closeup movie as the stimulus crosses the upper and lower vertical meridians.

34. M. I. Sereno, S. Pitzalis, A. Martinez, data not shown

35. Human V3A was originally defined as a thick strip of retinotopic (non-mirror image) cortex directly anterior to lower field-only V3 that contains upper as well as lower fields [R. B. Tootell et al., J. Neurosci. 17, 7060 (1997)]. Although the names "V3" and "V3A" were taken from macaque monkeys, the functional and anatomical properties of the human areas differ from their macaque monkey namesakes. The strong response to motion and the heavy myelination of human V3A [see figure 5 in M. I. Sereno, J. M. Allman, in The Neural Basis of Visual Function, A. Leventhal, Ed. (Macmillan, London, 1991), pp. 160172, and figure 8 in R. B. H. Tootell, J. B. Taylor, Cereb. Cortex 1995, 5, 39 (1995): labeled "possible/presumptive MT" in both instances] recall the direction selectivity and myelination of lower field-only macaque monkey $\mathrm{V} 3$ rather than macaque monkey $\mathrm{V} 3 \mathrm{~A}$. Owl monkeys also have a dorsal, heavily myelinated, direction-selective area, DM [J. M. Allman, J. H. Kaas, Brain Res. 100, 473 (1975)], that contains upper and lower fields like human V3A; but owl monkey DM directly adjoins V2 - like macaque V3 but unlike human V3A [see $(5,12)$ for comparison maps]. Different authors have subsequently published tentative and partly conflicting subdivisions of this region in humans [A. T. Smith, M. W. Greenlee, K. R. Singh, F. M. Kraemer, J. Hennig, J. Neurosci. 18, 3816 (1998); R. B. Tootell et al., Neuron 21, 1409 (1998); W. A. Press, A. A. Brewer, R. F. Dougherty, A. R. Wade, B. A. Wandell, Vis. Res. 41, 1321 (2001); R. B. Tootell, N. Hadjikhani, Cereb. Cortex 11, 298 (2001)]. To summarize, with a large V $3 A$ as a starting point, V3B is directly inferior to V3A, V7 is anterior to both V3A and V3B, and a two-part lateral occipital area in the expected location of V4d overlaps V3B completely. We are confident that our posterior focus overlaps the superior half of $\mathrm{V} 3 \mathrm{~A}$ and $\mathrm{V} 7$ but not $\mathrm{V} 3 \mathrm{~B}$ or the lateral occipital areas, and we are sure that our parietal focus lies completely anterior and superior to V7.
36. It is likely that the distractors also served as an (unbiased) probe of the attended-to region. Singleunit recording experiments in the macaque monkey LIP (22-24) have shown that when an animal is attending to a particular location, neurons with receptive fields at that location give an enhanced response to a probe stimulus there; a response that typically greatly exceeds the activity attributable to mere attention to that location on a blank screen.

37. Although peripheral attention is not necessary in standard phase-encoded retinotopic mapping experiments (the only task is continuous central fixation), it is unlikely that attention to the periphery can be completely suppressed. Maps derived from standard retinotopic mapping experiments therefore probably reflect a mix of "sensory" retinotopy and "attentional" retinotopy and also a different mix in different areas. With our task, there were weaker but significant responses in early retinotopic areas ( $\mathrm{V} 1$, $\mathrm{V} 2, \mathrm{~V} 3$, and $\mathrm{VP}$ ) that were retinotopically consistent in polar angle; that is, upper field (= red+blue) inferiorly and lower field (= green+blue) superiorly, and at the expected location in the eccentricity map. We set our significance thresholds conservatively (a steep sigmoidal function for the mapping between significance and color saturation); with a somewhat more lax criterion, these spotty responses at (just) the eccentricity corresponding to the target in V1/ V2/V3/VP become filled in.

38. In the parietal as well as the prefrontal cortex, most neurons signal the location of the target, even when the animal makes an anti-saccade [S. Funahashi, M. V. Chafee, P. S. Goldman-Rakic, Nature 365, 753 (1993); J. Gottlieb, M. E. Goldberg, Nature Neurosci. 2, 906 (1999)].

39. The orientation of the visual field map reported here (upper $\rightarrow$ anterior, lower $\rightarrow$ posterior) is roughly similar (once the cortex has been unfolded) to what was found in a mapping study of the LIP in anesthe- tized macaques [G. J. Blatt, R. A. Andersen, G. R. Stoner, J. Comp. Neurol. 299, 421 (1990); we made a surface reconstruction from the data shown in their figure 5]. One difference already noted is that the human area sits in a small sulcus slightly medial and posterior to the intraparietal sulcus instead of inside it. This recalls the situation with human MT, which typically sits in a small sulcus just posterior and ventral to the superior temporal sulcus instead of inside it, on its posterior bank. Finally, it has long been known that human V1 doesn't extend as far onto the lateral surface as it does in other primates. In each case, the presumed human homolog has moved radially outward (superiorly, posteriorly, and inferiorly) from the center of the lateral surface of the occipital lobe.

40. Retinotopically organized delay-period activity has been observed in a number of different brain struc tures in many different vertebrates. In monkeys, but also in frogs, a briefly presented stimulus can elicit retinotopically localized activity in the superior colliculus that persists over many seconds [D. Ingle, Science 188, 1033 (1975); L. E. Mays, D. L. Sparks, J. Neurophysiol. 43, 207 (1980); P. W. Glimcher, D. L. Sparks, Nature 355, 542 (1992)].

41. We thank R. Buxton, E. Wong, and L. Frank for their generosity with scan time, pulse sequences, and advice; C. Kemper for help with scanning; A. Dale for motion and retinotopic mapping stimulus code; $\mathrm{E}$. Awh, G. Boynton, A. Dale, B. Fischl, C. Fernety, M. Kutas, S. Hillyard, A. Liu, and E. Vogel for help and discussions; and G. Boynton for the use of his projection screen and frame. Supported by the Human Frontier Science program (E. Halgren), NIH grant NICHD22614 (M. Kutas), Office of Naval Research grant N00014-93-0942 (S. Hillyard), and NIMH grant MH25594 (A.M.).

22 June 2001; accepted 10 September 2001

\title{
CNS Synaptogenesis Promoted by Glia-Derived Cholesterol
}

\author{
Daniela H. Mauch, ${ }^{1}$ Karl Nägler, ${ }^{1,3}$ Stefan Schumacher, ${ }^{4}$ \\ Christian Göritz, ${ }^{1,3}$ Eva-Christina Müller, ${ }^{2}$ Albrecht Otto, ${ }^{2}$ \\ Frank W. Pfrieger ${ }^{1,3 *}$
}

The molecular mechanisms controlling synaptogenesis in the central nervous system (CNS) are poorly understood. Previous reports showed that a gliaderived factor strongly promotes synapse development in cultures of purified CNS neurons. Here, we identify this factor as cholesterol complexed to apolipoprotein E-containing lipoproteins. CNS neurons produce enough cholesterol to survive and grow, but the formation of numerous mature synapses demands additional amounts that must be provided by glia. Thus, the availability of cholesterol appears to limit synapse development. This may explain the delayed onset of CNS synaptogenesis after glia differentiation and neurobehavioral manifestations of defects in cholesterol or lipoprotein homeostasis.

The formation of synaptic contacts is a critical phase during brain development and plays a crucial role in long-term synaptic plasticity in the adult CNS, but the cell biological

${ }^{1}$ Synapse Group and 2 Protein Chemistry Group, MaxDelbrück-Center for Molecular Medicine, D-13092 Berlin, Germany. ${ }^{3}$ Max-Planck/CNRS Group, UPR 2396, Centre de Neurochimie, F-67084 Strasbourg France. ${ }^{4}$ Institute for Cell Biochemistry and Clinical Neurobiology, University of Hamburg, D-20246 Hamburg, Germany.

*To whom correspondence should be addressed. Email: fw-pfrieger@gmx.de mechanisms that mediate the assembly of the synaptic machinery are still poorly understood. A possible role of glial cells in CNS synaptogenesis was indicated by a series of studies (1-3) on rat retinal ganglion cells (RGCs), CNS neurons that can be highly purified (4) and cultured under defined gliafree conditions (5). The previous reports (13) showed that neurons formed few and inefficient synapses in the absence of glia and that glial cells induced the formation of numerous and highly efficient synapses without affecting neuronal survival, excitability, or 
neurite outgrowth. These effects were mediated by a soluble factor secreted by macroglial cells that has remained elusive so far.

To identify the glial factor, we pursued two experimental approaches. First, we fractionated glia-conditioned medium (GCM) (6) chromatographically, treated glia-free cultures of purified RGCs with elution fractions, and assayed their activity by whole-cell recordings of spontaneous excitatory postsynaptic currents (EPSCs) (7). The GCM-induced increase in synaptic activity was mediated by a heparin-binding factor that could be further purified by cation exchange chromatography $(8,9)$. Subsequent gel filtration (10) indicated that the factor migrated with large components of GCM (Fig. 1A). Second, we studied GCM-induced changes in the protein composition of membranes prepared from RGCs by two-dimensional gel electrophoresis (2-DE) (11-13). GCM treatment reliably caused the appearance of a new silverstained spot ( $n=3$ preparations) (Fig. 1B). Microsequencing by nanospray mass spectrometry $(14,15)$ showed that this spot contained apolipoprotein E (apoE) (Fig. 1C). In the $\mathrm{CNS}$, apoE is produced by macroglial cells (16-18). Therefore, it appeared likely that the apoE occurring in GCM-treated cells was of glial origin. Accordingly, immunoblots showed a lack of apoE in RGCs grown under glia-free conditions (19).

Initially, glia-derived apoE appeared to be a good candidate for the glial factor. It binds to heparin (20) and is part of glia-derived lipoprotein particles, whose size (21) falls in the same range that we had determined for the glial factor (Fig. 1A, inset). However, in RGC cultures treated with apoE (1 or 10 $\mu \mathrm{g} / \mathrm{ml})(22)$, the increase in EPSC frequency as compared to untreated controls averaged $0.8 \pm 0.3$-fold (mean $\pm \mathrm{SEM}, n=4$ cultures; Fig. 1D), showing that apoE was not the glial factor. Because apoE-containing lipoproteins serve as cholesterol carriers (23), we then tested whether cholesterol mimicked the effects of GCM. Treatment of RGC cultures with cholesterol $(10 \mu \mathrm{g} / \mathrm{ml})$ (22) increased the frequency of spontaneous EPSCs on average by $12 \pm 4-$ fold as compared to glia-free cultures and thus to a similar extent as treatment with GCM (15 \pm 4 -fold; $86 \pm$ $20 \%$ of GCM control; $n=13$ cultures) (Fig. 1D). We also tested whether this effect could be induced by other lipids contained in gliaderived lipoprotein particles $(21,24)$. However, treatment of neurons with phosphatidylcholine ( $0.4 \mu \mathrm{M} ; 2 \pm 1$-fold; $n=4$ cultures) or sphingomyelin $(0.4 \mu \mathrm{M} ; 0.3 \pm 0.2$-fold; $n=4)(22)$ did not significantly increase the frequency of spontaneous EPSCs as compared to untreated controls. At a higher con- centration $(2 \mu \mathrm{M})$, these lipids were toxic to purified RGCs. These results suggested that cholesterol complexed to large apoE-containing lipoproteins was the active component in GCM.

To test this hypothesis, we performed three experiments. First, we determined whether those gel filtration fractions with a strong effect on synaptic activity contained elevated levels of cholesterol and apoE (25). Our measurements showed that there was a close match between the synaptogenic activity of elution fractions and their combined content of cholesterol and apoE (Fig. 1E). Second, we tested whether lowering the cholesterol concentration in GCM would diminish its effect on synaptic activity. To produce low-cholesterol GCM (LC-GCM), we added mevastatin, an inhibitor of cholesterol synthesis (26), to glial cultures (27). Mevastatin $(10 \mu \mathrm{M})$ lowered the cholesterol concentration from $5.0 \pm 0.7 \mu \mathrm{g} / \mathrm{ml}$ in $\mathrm{GCM}$ to an undetectable level in LC-GCM ( $n=4$ cultures). In RGCs treated with LC-GCM, the spontaneous activity was strongly reduced as compared to the activity in cultures treated with normal GCM (Fig. 1F). This effect could be due to the drug-induced loss of a synaptogenic component other than cholesterol. However, supplementing LC-GCM with cholesterol $(10 \mu \mathrm{g} / \mathrm{ml})$ (LC-GCM+)
Fig. 1. Glia-derived cholesterol complexed to apoE-containing lipoproteins mediates the GCM-induced increase in synaptic activity. (A) Mean frequency of spontaneous EPSCS in RGC cultures treated with elution fractions obtained by gel filtration chromatography of prefractioned GCM. Inset: Calibration indicated a size range between $158 \mathrm{kD}(9.6 \mathrm{~nm})$ and $669 \mathrm{kD}(17 \mathrm{~nm})$ for the glial factor (gray shading, elution range of glial factor; arrows, molecular weight markers aldolase and thyroglobulin). (B) Silver-stained two-dimensional gels of membrane proteins from RGCs cultured without (left) and with (right) GCM (approximate range, molecular weight 28 to $45 \mathrm{kD}$, pl 4.5 to 5.5). Arrowhead, GCMinduced protein spot. (C) Identification of the protein marked in (B) as glia-derived apoE. The upper panel shows the nanoelectrospray mass spectrum of the peptide mixture from the trypsinized spot. Arrows indicate peptides selected for fragmentation. The lower panel shows the fragmentation spectrum of the doubly charged ion (mass-to-charge ratio, 620.34). Circles mark matches between peaks in the fragmentation spectrum and theoretical fragment masses for the identified apoE peptide (47). (D) Relative frequency of spontaneous EPSCs in individual RGC cultures treated with apoE (1 and $10 \mu \mathrm{g} / \mathrm{ml})$, cholesterol $(10 \mu \mathrm{g} / \mathrm{ml})$, or GCM, all compared to untreated control cultures. (E) Cholesterol (upper panel: thick line, left axis) and protein concentration (thin line, right axis) and apoE content (lower panel) in gel filtration fractions. In fraction 11, which had the strongest effect on synaptic activity as shown in (A), the combination of apoE and cholesterol content was maximal. (F) Lowering the cholesterol concentration in GCM (LC-GCM, $n=5$ cultures) reduced the GCMinduced increase in synaptic activity, and addition of cholesterol $(10 \mu \mathrm{g} / \mathrm{ml})$ to LC-GCM (LC-GCM+) fully restored the effect ( $n=2$ cultures). Inhibition of lipoprotein uptake by RAP $(50 \mu \mathrm{g} / \mathrm{ml}$ ) ( $n=4$ cultures) reduced the heparin eluate (HepElu)-induced increase in synaptic activity (31). In RAP-treated
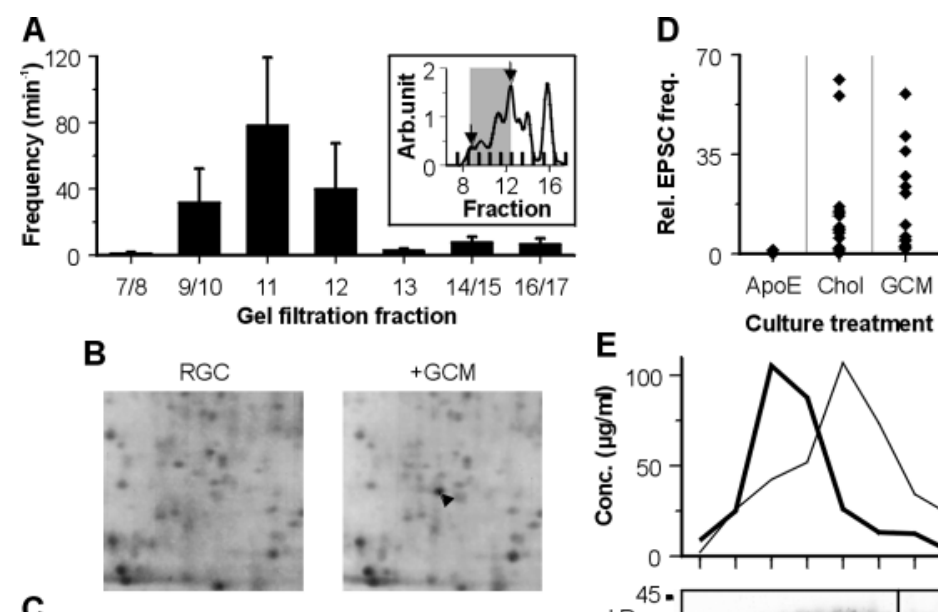

E


cultures, $6 \%$ of the RGCs tested (2 of 32 ) still showed strong synaptic activity. Open and solid columns represent mean values with and without these cells, respectively. Bars indicate SEM. 
fully rescued the strong increase in spontaneous synaptic activity ( $n=2$ cultures) (Fig. $1 \mathrm{~F})$, confirming that cholesterol was the active component. Third, we tested whether the GCM-induced increase in synaptic activity required apoE-binding receptors that mediate the uptake of lipoproteins (28). To accomplish this, we blocked members of the lowdensity lipoprotein (LDL) receptor family (29) with the receptor-associated protein (RAP) $(30,31)$. In RGCs treated with RAP and heparin-binding components of GCM, the spontaneous synaptic activity was reduced, as compared to the synaptic activity of controls treated with heparin eluate alone (Fig. 1F). In summary, five lines of evidence indicated that cholesterol produced by glial cells and secreted in apoE-containing lipoproteins was the glial factor. First, cholesterol alone strongly increased the frequency of spontaneous synaptic events, whereas other components of glia-derived lipoprotein particles did not affect synaptic activity. Second, there was a close match between the reported size of glial lipoproteins and the size of the synapse-promoting factor. Third, the level of activity in gel filtration fractions scaled with the combined content of cholesterol and apoE. Fourth, a drastic reduction of the cholesterol content in GCM by mevastatin eliminated its effects on synaptic activity. Fifth, inhibition of lipoprotein uptake reduced the
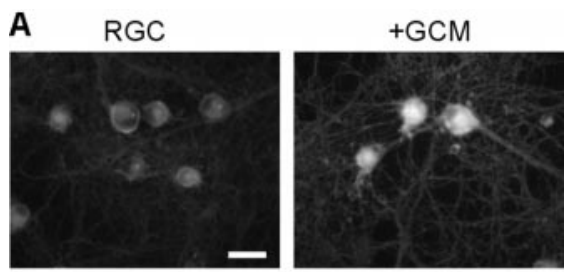

B

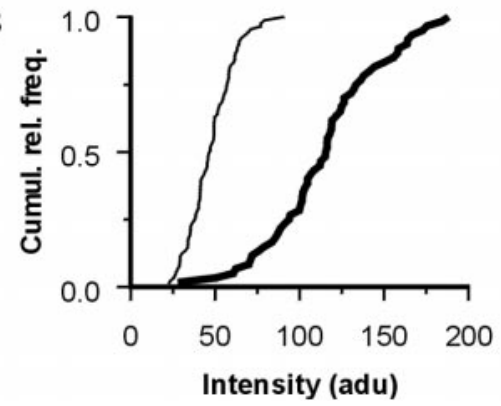

Fig. 2. Cholesterol content of cultured RGCs. (A) Fluorescence micrographs of purified RGCs cultured for 18 days in the absence of GCM (left) or for the last 7 out of 18 days in the presence of GCM (right) and stained with the cholesterol-binding antibiotic filipin. Both images were acquired under the same conditions. Scale bar, $30 \mu \mathrm{m}$. (B) Cumulative relative frequency distribution of background-corrected fluorescence intensities in somata of RGCs cultured without (thin line, $n=89$ cells) or with (thick line, $n=60$ cells) GCM pooled from three cultures $(P<0.001$, Student's $t$ test). effect of the heparin-binding fraction on synapses.

The identification of cholesterol as the glial factor prompted us to determine the

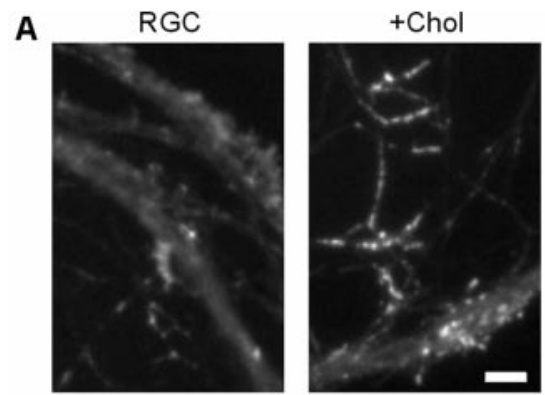

B
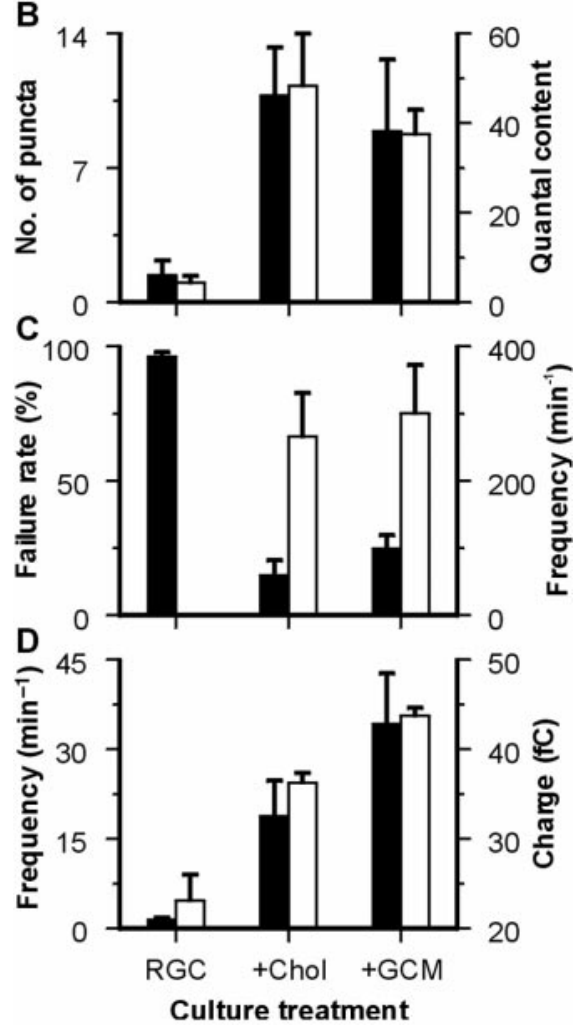

Fig. 3. Cholesterol increases the number and release efficacy of synapses in single RGCs. (A) Fluorescence micrographs showing RGCs cultured in the absence (left) or presence (right) of cholesterol and labeled by a synapsin I-specific antibody. Scale bar, $5 \mu \mathrm{m}$. (B) Number of synapsin- and GRIP-positive puncta per neuron (black columns, left axis) and quantal content of evoked EACs (white columns, right axis) in glia-free microcultures [ $n$ for RGC $=10$ cells (left axis)/4 cells (right axis)], in the presence of cholesterol $(10 \mu \mathrm{g} / \mathrm{ml}$, +Chol $25 / 16)$ or of GCM (+GCM 12/36). (C) Failure rate of evoked EACs (black columns, left axis) and frequency of asynchronous events (white columns, right axis) ( $n$ for RGC $=12,+$ Chol 20, +GCM 45) (D) Frequency (black columns, left axis) and charge transfer (white columns, right axis) of spontaneous EACs ( $n$ for RGC $=12 / 31,+$ Chol 22/408, +GCM 45/1402). Error bars indicate SEM. All cholesterol- and GCM-induced changes were statistically significant $(P<0.05$, Student's $t$ test). content and distribution of this important membrane component (32) in RGCs grown in the presence and absence of GCM. To accomplish this, we stained cultures with filipin, a fluorescent antibiotic that binds specifically to cholesterol $(33,34)$. In the absence of GCM, somata and neurites of RGCs showed detectable filipin staining with the background-corrected fluorescence intensity in neuronal somata averaging $48 \pm 1$ analogto-digital units (adu's) ( $n=89$ cells) (Fig. 2). In RGCs treated with GCM, however, filipin staining was much stronger, averaging $115 \pm$ 4 adu's in neuronal somata ( $n=60$ cells $)$ (Fig. 2). Thus, RGCs produced measurable levels of cholesterol, when cultured under defined conditions, and incorporated large amounts of glia-derived cholesterol when treated with GCM.

How does glia-derived cholesterol increase the frequency of spontaneous EPSCs? Previous studies show that GCM increases spontaneous synaptic activity by inducing the formation of new synapses and by enhancing the quantal size and efficacy of transmitter release $(2,3)$. To determine whether these effects were mediated by cholesterol, we studied microcultures of RGCs (35), in which synapse development can be quantified in individual neurons (2). The results showed that cholesterol essentially mimicked the previously reported effects of GCM on autapse development (2). Cholesterol raised the number of immunostained autapses by 8 -fold and the quantal content per neuron by 10 -fold, and thus acted similarly to GCM (Fig. 3, A and B). Analysis of neurite growth showed that these increases were caused by a direct synaptogenic effect rather than by a corresponding increase in neurite length (36). Furthermore, cholesterol increased the efficacy of transmitter release, as indicated by the low failure rate (Fig. 3C) and the nonlinear increase in asynchronous release (Fig. 3C), as compared to spontaneous release (Fig. 3D). Cholesterol also increased the charge transfer of spontaneous excitatory autaptic currents (EACs), but to a smaller extent than GCM (Fig. 3D), suggesting that additional glial signals control the quantal size.

How does cholesterol promote synapse formation? Cholesterol increased the number of synapsin I-positive puncta contained in neurites of RGCs (Fig. 3A) and thus mimicked a previously reported effect of GCM (2). We noticed, however, that cholesterol induced a stronger effect than GCM. In cholesterol-treated microcultures, the number of synapsin I-positive puncta per RGC was $69 \pm 23 \%(n=4$ cultures) larger than in GCM-treated controls. This effect was not restricted to synapsin I; cholesterol also induced $57 \pm 3 \%$ more synaptophysin-positive puncta than GCM ( $n=2$ cultures). Double immunostaining of cholesterol-treated cul- 
tures (37) revealed that the large majority of synapsin-positive puncta $(61 \pm 7 \%, n=9$ neurons) also contained synaptophysin, which indicates that these puncta represented synaptic vesicles or precursor material contained in presynaptic terminals or transport packets (38), respectively. These results suggested that cholesterol enhances the production of presynaptic components including synaptic vesicles (39) and release sites (40). Our previous finding (2) that GCM does not affect autapses immediately, but within 24 to 48 hours, suggests that the synaptogenic effect is mediated by such a slow multistep process.

Our study indicates that the ability of CNS neurons to form synapses is limited by the availability of cholesterol. RGCs cultured under serum- and glia-free conditions produced enough cholesterol to survive, to differentiate axons and dendrites (5), and to form a few immature synapses. Massive synaptogenesis requires large amounts of cholesterol and thus depends on cholesterol production by glial cells and its delivery via apoE-containing lipoproteins. Because cholesterol in the CNS is synthesized in situ rather than imported from blood (41-43), a link between synaptogenesis and glia-derived cholesterol could explain why most synapses in the developing brain are formed after the differentiation of macroglial cells $(3,44)$. Our results imply that genetic or age-related defects in the synthesis, transport, or uptake of cholesterol in the CNS $(30,45)$ may directly impair the development and plasticity of the synaptic circuitry.

\section{References and Notes}

1. F. W. Pfrieger, B. A. Barres, Science 277, 1684 (1997). 2. K. Nägler, D. Mauch, F. W. Pfrieger, J. Physiol. 533, 665 (2001).

3. E. M. Ullian, S. K. Sapperstein, K. S. Christopherson, B. A. Barres, Science 291, 657 (2001).

4. B. A. Barres, B. E. Silverstein, D. P. Corey, L. L. Y. Chun, Neuron 1, 791 (1988).

5. A. Meyer-Franke, M. R. Kaplan, F. W. Pfrieger, B. A. Barres, Neuron 15, 805 (1995).

6. GCM was harvested from primary glial cultures grown in a defined medium as described (2).

7. RGCs were purified from 6-day-old Sprague-Dawley rats by sequential immunopanning (4) and grown in a defined medium as described (2). Whole-cell recordings of spontaneous synaptic activity were performed and analyzed as described (1). To assay the effects of the glial factor on synaptic activity, spontaneous EPSCs were recorded from 5 to 10 RGCs cultured in the presence of column fractions. Before addition to RGC cultures, fractions were rebuffered to culture medium. To avoid indirect effects on neuronal survival, RGCs were cultured first for 1 week under defined conditions and then for at least 6 days in the presence of column fractions.

8. For heparin chromatography, GCM was centrifuged for $20 \mathrm{~min}$ at $3000 \mathrm{~g}$, and the supernatant was passed over a heparin column (HiTrap Heparin $5 \mathrm{ml}$, Amersham Pharmacia Biotech) using a fast protein liquid chromatography system (BioLogic, Biorad). Heparinbinding components were eluted with high salt and collected in a single fraction. For cation exchange chromatography, heparin eluate was rebuffered, passed over a cation exchange column (MonoS PC
1.6/5 column, SMART system, Amersham Pharmacia Biotech), and eluted by a salt gradient.

9. D. H. Mauch, S. Schumacher, F. W. Pfrieger, unpublished observation.

10. For gel filtration, the cation exchange column fraction with the strongest synaptogenic effect was concentrated (Microcon 10, Millipore) and passed over a gel filtration column (Superose 12 PC3.2/30 column, SMART system, Amersham Pharmacia Biotech). The column was calibrated with suitable gel filtration calibration kits (Amersham Pharmacia Biotech).

11. For 2-DE, carrier ampholyte urea isoelectric focusing (IEF) was combined with SDS-polyacrylamide gel electrophoresis (PAGE), as described (12). All reagents were obtained from WITA GmbH (Teltow, Germany). To prepare membrane fractions of RGCs, cells were washed twice with cold phosphate-buffered saline (PBS) and scraped off culture dishes into a lysis buffer containing (all Sigma) $25 \mathrm{mM}$ tris- $\mathrm{HCl}$ (pH 7.1), $50 \mathrm{mM} \mathrm{KCl}, 3 \mathrm{mM}$ EDTA, and protease inhibitor cocktail (1:10). Membranes were collected by ultracentrifugation for $30 \mathrm{~min}$ at $200.000 \mathrm{~g}$ and solubilized in the IEF sample buffer containing $7 \mathrm{M}$ urea, $2 \mathrm{M}$ thiourea, $25 \mathrm{mM}$ tris- $\mathrm{HCl}(\mathrm{pH} 7.1), 50 \mathrm{mM}$ $\mathrm{KCl}, 3 \mathrm{mM}$ EDTA, $2.9 \mathrm{mM}$ benzamidin, $2.1 \mu \mathrm{M}$ leupeptin, $70 \mathrm{mM}$ dithiothreitol, $2.5 \%$ carrier ampholytes (pH 2 to 4, Servalyt), and 4\% CHAPS. SDS-PAGE was performed in $15 \%$ acrylamide gels $(0.7 \mathrm{~mm}$ thick) with IEF gels used as stacking gels. Proteins were visualized by silver staining as described (13).

12. J. Klose, U. Kobalz, Electrophoresis 16, 1034 (1995).

13. H. Blum, H. Beier, H. I. Gross, Electrophoresis 8, 93 (1987).

14. For protein identification, spots were excised, destained, reduced, S-alkylated, and in-gel digested with trypsin. The resulting peptide mixtures were desalted and analyzed by nanoelectrospray mass spectrometry. Mass spectra were recorded on a hybrid quadrupole time-of-flight mass spectrometer (Q-Tof, Micromass, Manchester, UK). Proteins were identified by the peptide sequence tag method (15).

15. M. Wilm et al., Nature 379, 466 (1996).

16. J. K. Boyles, R. E. Pitas, E. Wilson, R. W. Mahley, J. M. Taylor, J. Clin. Invest. 76, 1501 (1985)

17. G. Stoll, H. W. Meuller, B. D. Trapp, J. W. Griffin, Glia 2, 170 (1989).

18. U. Beffert et al., Brain Res. Rev. 27, 119 (1998).

19. D. H. Mauch, C. Göritz, F. W. Pfrieger, unpublished observation.

20. R. W. Mahley, K. H. Weisgraber, T. L. Innerarity, Biochim. Biophys. Acta 575, 81 (1979).

21. M. J. LaDu et al., J. Neurochem. 70, 2070 (1998).

22. Recombinant human apoE3 (Calbiochem), cholesterol (from ethanol stock, $n=13$ cultures; or complexed to methyl- $\beta$-cyclodextrin, $n=5$; Sigma), stearoylsphingomyelin (semisynthetic, from ethanol stock; Sigma) or phosphatidylcholine (synthetic, with oleic acid and palmitic acid; from ethanol stock; Sigma) were added directly to 1-week-old RGC cultures, and synaptic activity was assayed after 1 week of treatment. Ethanol (final concentration $=0.1 \%$ ) had no effect on synaptic activity (46). Control cultures were treated with GCM as described (2).

23. R. W. Mahley, Science $\mathbf{2 4 0} 622$ (1988).

24. J. Ito, L. Y. Zhang, M. Asai, S. Yokoyama, J. Neurochem. 72, 2362 (1999).

25. Cholesterol and protein concentrations were measured by standard assays (Amplex Red Cholesterol Assay Kit, Molecular Probes; and BCA assay, Pierce) according to the manufacturer's instructions using human LDL (Calbiochem) and bovine serum albumin (Sigma) as the standard, respectively. ApoE was detected in column fractions by immunoblotting with a rabbit serum (1:1000; gift of T. E. Willnow).

26. A. Endo, Y. Tsujita, M. Kuroda, K. Tanzawa, Eur. J. Biochem. 77, 31 (1977)

27. To obtain LC-GCM, mevastatin ( $10 \mu \mathrm{M}$ from $10 \mathrm{mM}$ ethanol stock) was added to glial cultures for 1 week, during which LC-GCM was collected three times. Before addition to RGC cultures, residual mevastatin was removed by rebuffering LC-GCM to neuronal culture medium (NAP10 columns, Amersham Pharmacia Biotech). Rebuffering itself or addition of mevastatin $(10 \mu \mathrm{M})$ to normal GCM before rebuffering did not inhibit the GCM-induced effects on synaptic activity (46). To determine the cholesterol content in GCM and LC-GCM, medium components that interfered with the enzyme-based assay (25) were removed by methanol/chloroform extraction.

28. M. S. Brown, J. L. Goldstein, Science 232, 34 (1986). 29. J. Herz, Neuron 29, 571 (2001).

30. T. E. Willnow, Biol. Chem. 379, 1025 (1998).

31. To block neuronal lipoprotein uptake by RAP, eluate from the heparin column was used as a control instead of GCM to avoid strong dilution of the inhibitor. Heparin eluate $(100 \mu l)$ was rebuffered to culture medium and added to RGC cultures during regular medium change. Glutathione $S$-transferase (GST)-tagged RAP ( $50 \mu \mathrm{g} / \mathrm{ml}$; gift of T. E. Willnow, MDC) or GST $(25 \mu \mathrm{g} / \mathrm{ml})$ was added every 12 hours over the entire period of treatment ( 6 days). GST did not inhibit the effect of heparin eluate (46).

32. K. Simons, E. Ikonen, Science 290, 1721 (2000).

33. A. W. Norman, R. A. Demel, B. de Kruyff, L. L. van Deenen, J. Biol. Chem. 247, 1918 (1972).

34. RGC cultures were fixed with $4 \%$ paraformaldehyde in PBS for 30 min, washed twice with PBS, incubated for 2 hours with filipin $(40 \mu \mathrm{g} / \mathrm{ml})$ [in PBS, from ethanol stock $(20 \mathrm{mg} / \mathrm{ml})$; Sigma], and washed twice with PBS. Filipin fluorescence was examined on the same imaging setup as described (2), using monochromatic light of $356 \mathrm{~nm}$ for excitation and a suitable filter set (set no. 2, Carl Zeiss). The fluorescence intensity of neuronal somata and of the cell-free background was measured semiautomatically by a custom-written Labview routine (National Instruments, Munich) with an observer outlining manually respective areas in each image. Fluorescence intensity is given in adu's.

35. Preparation of RGC microcultures, immunocytochemical staining of autapses, electrophysiological recordings of spontaneous, evoked and asynchronous autaptic currents, and data analysis were performed as described (2).

36. To assess possible effects of cholesterol and GCM on neurite length, we determined for each neuron immunolabeled by synapse markers the percentage of the microisland area (manually outlined, diminished by soma area) that was covered by neurites. Neurites were reliably detected by image segmentation (threshold: mean intensity $+10 \times$ SD of neurite-free background). Cholesterol ( $31 \pm 1 \%, n=42$ cells) and $\operatorname{GCM}(22 \pm 1 \%, n=29$ cells) increased the neurite coverage by 1.3 fold and by 0.9 fold as compared to coverage of untreated cultures $(24 \pm 2 \%, n=24$ neurons), respectively, and thus to a much smaller extent than the synapse number.

37. For double immunostaining of synaptic vesicles, we used antibodies against synapsin I (polyclonal, 1:200, Synaptic Systems, Göttingen, Germany) and against synaptophysin (monoclonal, clone SVP-38, 1:200, Sigma).

38. S. E. Ahmari, J. Buchanan, S. J. Smith, Nature Neurosci. 3, 445 (2000).

39. A. Thiele, M. J. Hannah, F. Fahrenholz, W. B. Huttner, Nature Cell Biol. 2, 42 (2000).

40. T. Lang et al., EMBO J. 20, 2202 (2001).

41. J. J. Kabara, Prog. Brain Res. 40, 363 (1973)

42. M. Danik, D. Champagne, C. Petit-Turcotte, U. Beffert, J. Poirier, Crit. Rev. Neurobiol. 13, 357 (1999).

43. J. M. Dietschy, S. D. Turley, Curr. Opin. Lipidol. 12, 105 (2001).

44. F. W. Pfrieger, B. A. Barres, Curr. Opin. Neurobiol. 6, 615 (1996)

45. R. V. Farese, J. Herz, Trends Genet. 14, 115 (1998). 46. D. H. Mauch, F. W. Pfrieger, unpublished observation.

47. With the indicated sequence tag Gly-Ala-Gly-LeuAsn, the protein was identified by database search as apoE. Analysis of two other peptides indicated in the upper panel of Fig. 1C confirmed this result.

48. We thank I. Haupt and J. Klewer for technical assistance, T. E. Willnow for the gift of reagents, and G. Lewin and T. E. Willnow for discussions and comments on the manuscript. Supported by grants from the Deutsche Forschungsgemeinschaft to F.W.P. (SFB 515) and to S.S. (SFB 545).

26 July 2001; accepted 21 September 2001 